\title{
Histidine modification and mutagenesis point to the involvement of a large conformational change in the mechanism of action of phage lambda lysozyme
}

\author{
Christine Evrard, Jacques Fastrez, Patrice Soumillion \\ Université Catholique de Louvain, Laboratoire de Biochimie Physique et des Biopolymères, Place L. Pasteur 1-1B, B-1348 Louvain-la- \\ Neuve, Belgium
}

\begin{abstract}
Phage lambda lysozyme $(\lambda \mathrm{L})$ is structurally related to other known lysozymes but its mechanism of action is different from the classical lysozyme mechanism, acting as a transglycosidase rather than a hydrolase. As two conformations have been revealed by the crystal structure, we investigated the effect of mutating and modifying a histidine located near to or far from the active site in the respective closed and open conformations. Whereas its asparagine mutation has little or no effect on activity, its $N$-carbethoxylation inactivates the enzyme. This provide further evidence for the involvement of the closed conformation and for the need of conformational mobility in $\lambda \mathrm{L}$ function.
\end{abstract}

Key words: Lysozyme; Diethyl pyrocarbonate; Functional motion

Abbreviations: DEPC, diethyl pyrocarbonate; $\lambda \mathrm{L}$, T4L, HEWL, respectively the lysozymes of lambda, T4 and hen egg white

\section{INTRODUCTION}

Obviously, proteins cannot be reduced to rigid and organized functional structures. Flexibility and conformational changes often play critical roles for protein function [1]. Especially in the case of enzymatic catalysis, conformational mobility can help an enzyme to adapt its active site along the reaction pathway such that all the steps, from substrate binding to product release, are optimized. A great variety of internal motions occurring on different time scales and amplitudes have been described and some of them have been shown to be involved in the mechanism of action of enzymes. Large conformational changes have been reported upon substrate binding, particularly the hinge-bending motion often observed for enzymes made of two domains. More subtle conformational events can also accompany the different steps of the catalytic cycle or the mechanism of allosteric regulation.

In this study, we investigate the hypothesis of a functional motion possibly occurring for the lambda lysozyme $(\lambda \mathrm{L}) . \lambda \mathrm{L}$ is implicated in the degradation of the peptidoglycan of Escherichia coli in the final stage of the infection cycle. This 158 residue protein has been purified and characterized [2-5]. Its three-dimensional structure has been determined recently [6] and has confirmed the prediction that $\lambda \mathrm{L}$ is evolutionarily related to other viral lysozymes even though the sequences are almost unrelated, $\lambda \mathrm{L}$ sharing only $18 \%$ sequence identity with the well-known lysozyme of phage T4 (T4L) [7]. The enzyme is made up of two domains between which the active site cleft of the enzyme is formed. Interestingly, the crystallo-graphic data revealed two distinct conformational states for $\lambda \mathrm{L}$ but, instead of a hinge-bending motion between the two domains as observed for T4L [8-10], the change comes from a peptide segment in the C-terminal domain of the enzyme that either opens or closes the active site cleft. This observation is probably not a crystallization artifact as conformational heterogeneity in solution has also been described from ${ }^{19} \mathrm{~F}$ NMR for a derivative of $\lambda \mathrm{L}$ labelled with trifiuoromethionine [11].

The $\lambda \mathrm{L}$ structure also confirmed the position of Glu-19, the essential glutamic acid common to all known lysozymes, in the active site cleft whereas there is apparently no second catalytic residue such as Asp-20 for T4L or Asp-52 for hen egg white lysozyme (HEWL). Moreover, the mechanism of $\lambda \mathrm{L}$ has been shown to be different from that of the intensively investigated HEWL and T4L (for reviews on lysozymes, see [12-15]): $\lambda \mathrm{L}$ is not a hydrolase but a transglycosidase, the hydroxyl function on the C6 of the muramic acid of the mur-opeptide replaces water as a nucleophile in the reaction with the oxycarbocation intermediate; the product is a 1,6-anhydro-muropeptide [16-18]. The same 1,6-anhydro-muropeptide is formed by the soluble lytic transglycosidase (Slt70) of E. coli [19], a $70 \mathrm{kDa}$ enzyme containing a catalytic domain showing significant structural similarity 
with the T4 lysozyme despite the lack of detectable sequence similarity [20]. Interestingly, the 'catalytic' aspartate is also absent in Slt70 [21], a difference shared by $\lambda \mathrm{L}$ that could be related to a different mechanism of cleavage of the $\beta$-1,4-glycosidic bond. Nevertheless, this aspartate is also lacking in several other lysozymes with hydrolase activity [15] and the origin of the change in mechanism between the $\lambda \mathrm{L}$ and Slt70 transglycosidases and the other lysozymes remains unexplained.

To test the hypothesis of a functional motion, we focused our attention on a histidine residue (His-137) located at a surface position distant from the active site cleft in the open conformation and which is found in a rather buried environment in the vicinity of the active site Glu-19 in the closed conformation. The effects of chemical modification with diethyl pyrocarbonate (DEPC) and of mutation to asparagine were analyzed. The two other histidines of $\lambda \mathrm{L}$ (His-31 and His-48) were also mutated in order to unambiguously address the chemical modification of His-137. Whereas the $\mathrm{H} 31 \mathrm{~N}-\mathrm{H} 137 \mathrm{~N}$ mutant behaves essentially as the wild type enzyme, the $N$ carbethoxylation of His-137 by DEPC appears to inactivate the wild type enzyme. These results support the hypothesis that the conformational mobility observed in the crystal structures of $\lambda \mathrm{L}$ is an intrinsic functional property of $\lambda \mathrm{L}$ in solution. Implications for the mechanism of action are discussed.

\section{MATERIALS AND METHODS}

\subsection{Materials}

Restriction enzymes were purchased from Boehringer, Eurogentec (Belgium) and Gibco BRL. Calf intestinal alkaline phosphatase, T4 DNA ligase and T4 polynucleotide kinase were purchased from Gibco BRL. All other reagents were of analytical grade. Oligonucleotides were purchased from Eurogentec:

- (I) 5'-ATAACCATCATTTCTGG-3'

- (II) 5'-GCGAGGGTTATCGGAGT-3'

- (III) 5'-CAGAAAACTAGAAATAATGGTTATGACGT-3'

- (IV) 5'-CATAACCATTATTTCTAGTTTTCTGACGT-3'

- (V) 5'-TCACTGCCGGGCGCTGGTTATGGTCAGTTC-GAGAATAAGGCTGAC-3

- (VI) 5'-TGCAGGGTCGACGGATCCTCGGGAATTCA-T-3'

\subsection{Genetic engineering methods}

The lambda $R$ gene encoding the lysozyme has been cloned with a synthetic ribosome binding site in a pPlc236 vector [22] to give pLJ0516 where it is expressed at a high level from the PL promoter under the control of the thermolabile $\mathrm{C}_{\mathrm{I}} 857$ repressor [23].

The H31D and H48N mutants were constructed by oligonucleotide -directed mutagenesis following the phosphorothioate procedure [24] with primers I and II respectively.

The H31N and the H31N-H137N mutants were made by cassette mutagenesis. For the H31N mutation, we took advantage of two closely spaced AatII sites flanking the H31 codon. The plasmid pLJ0516 was digested by AatII to excise a $31 \mathrm{bp}$ fragment. The aga-rose-purified large fragment was dephosphorylated with calf intestinal alkaline phosphatase (CIAP). A mixture of two complementary oligonucleotides, III and IV, bearing the required mutations was phos-phorylated with T4 polynucleotide kinase. After enzyme inactivation at $70^{\circ} \mathrm{C}$ and hybridization, the synthetic duplex was ligated with the large fragment by T4 DNA ligase. To select the plasmid (pPS07) bearing the insert in the correct orientation, a silent mutation introducing a stop codon in the incorrect orientation was introduced. Upon induction on small cultures, lysozyme was detected by SDS-PAGE electrophoresis.

For unknown reasons, the H137N mutation could not be made by oligonucleotide-directed extension mutagenesis. To overcome the problem, we took advantage of the existence of a NciI site in the $R$ gene near the H137 codon. The plasmid encoding the double mutant was reconstructed in a three fragment ligation: (1) a large fragment $(4.3 \mathrm{kbp}$ ) was derived from $B g l \mathrm{II}$ and SalII digestion of pPS08, a plasmid identical to pPS07 except that the HindIII site upstream of the $R$ gene is replaced by BglII; (2) a 395 bp fragment was derived from NciI restriction of the small fragment obtained after $\mathrm{BglII}-\mathrm{SalI}$ restriction; (3) a $125 \mathrm{bp}$ fragment, which introduces the H137N mutation and removes the SmaI and NciI sites of the polylinker, was generated by PCR with primers V and VI and restricted with SalI and NciI. The 395 and 125 bp fragments are phosphorylated with T4 kinase. After ligation and transformation, the expected plasmid, pPS10, is found by restriction with BglII, SalI and SmaI. 


\subsection{Purification and characterization of the enzymes}

The wild type and mutant $\lambda \mathrm{L}$ were expressed in $E$. coli strain M5219 harboring the corresponding plasmids following a protocol described previously that strongly reduces the heat shock-induced proteolysis in vivo [25]. The wild type $\lambda \mathrm{L}$ and the H31D, H31N-H137N mutants were purified as described previously [23]. The H48N mutant enzyme was found as inclusion bodies and was rena-tured before purification as described previously [7].

Activities and stabilities to reversible thermal denaturation were measured as described previously $[23,26]$. Activity-pH profiles were obtained at an ionic strength of $20 \mathrm{mM}$. Reversible denaturations were followed in 100 $\mathrm{mM}$ phosphate buffer, $\mathrm{pH}$ 7.0.

Chemical modification of the histidines was performed with DEPC [27]. Solutions of wild type or mutant $\lambda \mathrm{L}$ of known concentration were prepared by measuring their $A_{280}$ and taking an extinction coefficient of $30000 \mathrm{M}^{-1}$ $\mathrm{cm}^{-1}$ [26]. The chemical modification was followed by measuring the absorbance change in the far UV and the effect on activity. $20 \mu \mathrm{l}$ of a $0.4 \mathrm{M}$ solution of DEPC in acetoni-trile was added to $2 \mathrm{ml}$ of $10^{-5} \mathrm{M}$ solutions of the enzymes in $50 \mathrm{mM}$ phosphate or MES buffer at $\mathrm{pH}$ 6.0. The absorbance was followed at $242 \mathrm{~nm} .16 \mu 1$ of $0.4 \mathrm{M}$ DEPC in acetonitrile was added to $1 \mathrm{ml}$ of $6 \times 10^{-7} \mathrm{M}$ enzyme in $50 \mathrm{mM}$ phosphate buffer at $\mathrm{pH} 6.0$. Aliquots of this solution were taken to measure the residual activity as a function of time. For the H31N-H137N mutant, measurements were performed in bis-Tris ( $\mathrm{pH}=7.5,7.0$ and 6.5) or MES ( $\mathrm{pH}=6.5,6.0$ or 5.5) buffers. The reversibility of the modification was measured by adding $50 \mu \mathrm{l}$ of hydroxylamine at a final concentration of 0.1 $\mathrm{M}$ to an enzyme solution whose residual activity in the course of inhibition had reached approximately $5 \%$. The evolution of the lytic activity was then followed by measurements on aliquots as a function of time.

Fig. 1. $p H$ dependence of the lytic activity observed for wild type (squares), H31D (triangles), H31N-H137N (circles) and H48N (diamonds) lysozymes. Activities were obtained by measuring the lysis rate of suspensions of EDTA-sensitized E. coli cells (decrease of the suspension turbidity at $570 \mathrm{~nm}$ ). Rates are expressed in relative units; 100 units correspond to the wild type maximum activity observed at $\mathrm{pH} 7$.

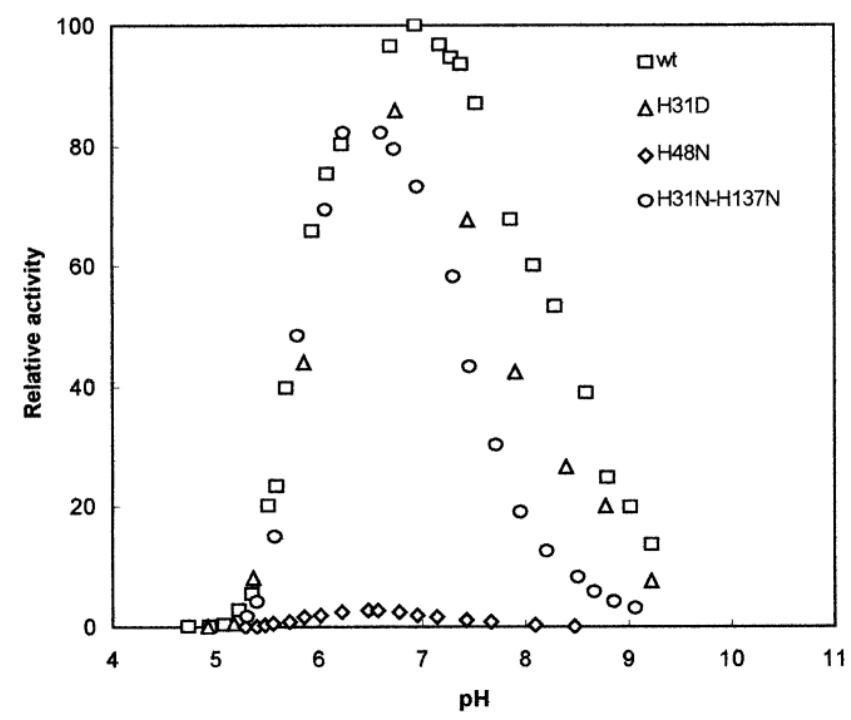

\section{RESULTS}

\subsection{Enzymatic activity and stability of wild type and mutants $\lambda L$}

The lytic activity of these enzymes on EDTA-sensitized $E$. coli cells was measured as a function of $\mathrm{pH}$ at an ionic strength of $20 \mathrm{mM}$. As shown in Fig. 1, the maximal activities of the H31D and the H31N-H137N mutants were very similar to that of the wild type enzyme. The H48N mutation reduced the maximal activity to $3 \%$ of the wild type.

The stability of mutant enzymes to reversible denaturation was measured at $\mathrm{pH} 7.0$ by following the fluorescence of the protein as a function of temperature. Sigmoidal curves were obtained for all the mutants. An identical loss of fluorescence intensity $(50 \%)$ was observed upon unfolding of wild type, H31D and H31N-H137N enzymes. This information, together with the catalytic activity measurements, indicates that these two mutant enzymes are 
properly folded. A lower fluorescence decrease was observed upon unfolding of the H48N mutant (40\%). The $T_{\mathrm{m}}$ and $\Delta H_{D}\left(T_{\mathrm{m}}\right)$ values were extracted from the fluorescence curves and are given in Table 1 together with the free energy of denaturation calculated at $25^{\circ} \mathrm{C}$ by the method described previously [26].

\subsection{Chemical modification with DEPC}

On incubation with $0.4 \mathrm{mM}$ DEPC at $\mathrm{pH}$ 6.0, the wild type $\lambda \mathrm{L}$ loses its activity with a half-time of about $7 \mathrm{~min}$. The inactivation may result from the chemical modification of one or several histidines as DEPC is a reagent designed to modify these residues selectively [27]. $N$-Carbethoxylation of a histidine is accompanied by a spectral change in the far UV: the absorbance at $242 \mathrm{~nm}$ increases $\left(\Delta \varepsilon=3200 \mathrm{M}^{-1} \mathrm{~cm}^{-1}\right)$. However, this reagent is not totally specific for histidines: lysines, tyrosines and cysteines are susceptible to modification giving products of high, medium or very low stability [28]. $O$-Carbethoxylation of tyrosine is detectable by its effect on absorbance at $278 \mathrm{~nm}\left(\Delta \varepsilon=-1310 \mathrm{M}^{-1} \mathrm{~cm}^{-1}\right)$ [29]. The carb-ethoxylation of histidine can be reversed by incubation with $20 \mathrm{mM}$ hydroxylamine while that of tyrosines or lysines is not.

In order to determine the origin of the DEPC inactivation of $\lambda \mathrm{L}$, we have run chemical modification experiments on several mutants, and followed the spectral changes and the effects on activities.

The H31N-H137N mutant contains only one remaining histidine (His-48). On incubation of this enzyme with 0.4-4 mM DEPC, an exponential decrease in activity was observed. The rate constant of the reaction was determined by fitting the residual activity $A_{\Gamma}$ with the following equation:

$$
A_{\mathrm{r}}=A_{\mathrm{i}} \mathrm{e}^{-k_{1} t}
$$

A second order rate constant was obtained by dividing $k_{1}$ by the DEPC concentration:

$$
k_{1}=k_{2}[\mathrm{DEPC}]
$$

The rate constants of inactivation were measured at several pHs. They increased exponentially from $0.04 \mathrm{M}^{-1} \mathrm{~s}^{-1}$ at $\mathrm{pH} 5.5$ to $2.3 \mathrm{M}^{-1} \mathrm{~s}^{-1}$ at $\mathrm{pH}$ 7.5. As no levelling off was observed at $\mathrm{pH} 7.5$ (data not shown), the $\mathrm{p} K_{\mathrm{a}}$ of the modified residue must be above 7.5. The inactivation could not be reversed by incubation with $100 \mathrm{mM} \mathrm{NH}_{2} \mathrm{OH}$. When the modification was followed at $242 \mathrm{~nm}$ at $\mathrm{pH} 7$ and $8^{\circ} \mathrm{C}$ no absorbance increase was detected (the low temperature was used to avoid a slow precipitation of the enzyme). All these results show that His-48 cannot be modified; the inactivation is most probably a consequence of the modification of the lysine side chains or of the $\alpha$-amino function.

Table 1: Thermodynamic parameters for the reversible thermal denaturation of wild type and mutant lysozymes

\begin{tabular}{lcll}
\hline Enzyme & $\mathrm{T}^{\mathrm{a}}{ }_{\mathrm{m}}\left({ }^{\circ} \mathrm{C}\right)$ & $\Delta \mathrm{H}_{\mathrm{D}}\left(\mathrm{T}_{\mathrm{m}}\right)^{\mathrm{b}}(\mathrm{kcal} / \mathrm{mol})$ & $\Delta \mathrm{GD}(25)^{\mathrm{C}}(\mathrm{kcal} / \mathrm{mol})$ \\
\hline$\lambda \mathrm{L}_{\mathrm{wt}}$ & 52.3 & 113.3 & 7.1 \\
$\lambda \mathrm{L}_{\mathrm{H} 31 \mathrm{D}}$ & 46.1 & 99.8 & 5.5 \\
$\lambda \mathrm{L}_{\mathrm{H} 31 \mathrm{~N}-\mathrm{H} 137 \mathrm{~N}}$ & 54.3 & 107 & 8.9 \\
$\lambda \mathrm{L}_{\mathrm{H} 48 \mathrm{~N}}$ & 30.1 & 60.8 & 2.1 \\
\hline${ }^{\mathrm{a}}$ Temperature of half denaturation (experimental). ${ }^{\mathrm{b}}$ Enthalpy of denaturation at $T_{\mathrm{m}}$ (experimental). ${ }^{{ }^{\circ}}$ Tree energy of denaturation at $25^{\circ} \mathrm{C}$ \\
(calculated as described in[30]).
\end{tabular}

The effect of H137 modification was analyzed by incubation of the H31D mutant at pH 6.0 with DEPC: an absorbance increase $\left(\Delta A_{\mathrm{obs}}\right)$ was observed at $242 \mathrm{~nm}$ as a function of time; it can be fitted with the following equation:

$$
\Delta A_{\mathrm{obs}}=\Delta A \cdot\left(1-\mathrm{e}^{-k_{1} t}\right)
$$

which is used with Eq. 2 to determine the second order rate constant: $k_{2}=2.3 \mathrm{M}^{-1} \mathrm{~s}^{-1}$ at $\mathrm{pH}$ 6. Dividing the $\Delta A$ by the enzyme concentration gives a $\Delta \varepsilon_{242}=3250 \mathrm{M}^{-1} \mathrm{~cm}^{-1}$. This value is indicative of the modification of one histidine. As His-48 is not modified, this reflects the modification of H137. The effect of this modification on activity was also determined: the enzyme was inactivated with a rate constant $k_{2}=3.8 \pm 0.5 \mathrm{M}^{-1} \mathrm{~s}^{-1}$. The inactivation can be partially reversed $(40 \%)$ by incubation with $\mathrm{NH}_{2} \mathrm{OH}$. This lack of full reactivation comes 
most probably from the combination of the rapid reversible modification of His-137 and the slow irreversible modification described for the $\mathrm{H} 31 \mathrm{~N}-\mathrm{H} 137 \mathrm{~N}$ mutant. The effect of $\mathrm{H} 31$ modification was estimated by analysis of the modification of the wild type $\lambda \mathrm{L}$ at $\mathrm{pH}$ 6.0. It is known that H137 is modified but not H48. If H31 is also modified, the absorbance increase at $242 \mathrm{~nm}$ in the presence of DEPC should follow a bi-exponential curve according to the following equation:

$$
\Delta A_{\text {obs }}=\Delta A \cdot\left(1-0.5 \mathrm{e}^{-k_{1 \mathrm{a}} t}+0.5 \mathrm{e}^{-k_{1 \mathrm{~b}} t}\right)
$$

where $k_{1 \mathrm{a}}$ and $k_{1 \mathrm{~b}}$, are the first order rate constants of modification of His-31 and His-137. Second order rate constants are obtained by dividing them by the DEPC concentration (Eq. 2). For fitting the observed absorbance change to Eq. $4, k_{2 . \mathrm{H} 137}$ is fixed at $2.3 \mathrm{M}^{-1} \mathrm{~s}^{-1}$. From the other parameters obtained in the fitting and the known enzyme concentration, the following values are obtained: $k_{2 . \mathrm{H} 31}=1.26 \mathrm{M}^{-1} \mathrm{~s}^{-1}$ and $\Delta \varepsilon_{242}=5400 \mathrm{M}^{-1} \mathrm{~cm}^{-1}$, i.e. 2700 $\mathrm{M}^{-1} \mathrm{~cm}^{-1}$ per histidine. No decrease in absorbance at $278 \mathrm{~nm}$ is observed indicating that tyrosines are not modified under these conditions. The wild type enzyme is also inactivated by DEPC; the activity decays according to a single exponential from which a second order rate constant can be obtained: $k_{2}=3.9 \pm 0.7 \mathrm{M}^{-1} \mathrm{~s}^{-1}$. This value is close to that observed with the H31D mutant. From these results, it appears that H31 can be modified but that its modification does not inactivate the enzyme. Again the inactivation can be partially (40\%) reversed by hydroxylamine treatment.

\section{DISCUSSION}

In the recently determined structure of $\lambda \mathrm{L}$ [6] each histidine of $\lambda \mathrm{L}$ appears to be situated in a different environment (Fig. 2). His-31 is completely solvent exposed and located in a turn relatively far ( $12 \AA ̊$ ) from the essential Glu-19 in the active site cleft. Its environment is identical in both enzyme conformations. Therefore, it is not surprising that its modification and mutation does not significantly affect the enzyme activity. The rate constant of modification is consistent with literature values that are in the range of $0.4-12.5 \mathrm{M}^{-1} \mathrm{~s}^{-1}$ around $\mathrm{pH} 7$ for accessible histidines residues [27]. On the surface of $\lambda \mathrm{L}$, His-31 is close to the negatively charged Asp-22 ( $~ 5$ Á). Therefore, the effect of the H31D mutation on the protein stability is consistent with a weak destabilization upon introduction of an additional negative charge. Moreover, the $\mathrm{pH}$ dependence of the difference in free energy of unfolding between $\lambda \mathrm{L}_{\mathrm{wt}}$ and $\lambda \mathrm{L}_{\mathrm{H} 31 \mathrm{D}}$ revealed that the $\mathrm{p} K_{\mathrm{a}}$ of His-31 is slightly higher than normal $(\sim 7.2$, data not shown), indicating a stabilizing interaction between His-31 and Asp-22.

The $\beta$ domain of the enzyme contains His- 48 , located in a coiled structure just after a $\beta$-strand. As for His-31, the environment of His-48 is identical in both enzyme conformations. This residue is completely buried in a hydrophobic pocket and its $\mathrm{p} K_{\mathrm{a}}$ has been shown to be very low ( $\leq 2.7$ [30]). Hence, it is not surprising that His-48 cannot be modified by DEPC. The reduced activity of the H48N mutant is most probably due to a perturbation affecting the structure of the $\beta$ domain as suggested by the loss of around $5 \mathrm{kcal} / \mathrm{mol}$ calculated for the His- 48 mutation (Table 1).

As shown in Fig. 2, the large conformational change revealed by the crystallographic study [6] involves residue 137. In the crystal lattice, the enzyme was found in two distinct states, an open conformation and a closed one. In the open structure, His-137 is part of the amino-terminal end of the last $\alpha$-helix, well accessible to the solvent and relatively far away from the active site cleft (about 18 A from the essential Glu-19). The shortest possible

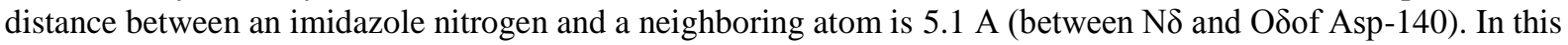
environment, His-137 should react easily with DEPC without affecting the enzymatic activity. In the closed structure, this helix is partially disrupted and residues 128-138 have moved towards the active site cleft, the distance between Glu-19 and His-137 being reduced to about 9 A. The imidazole ring is partially buried and is poorly accessible for $N$-carbethoxylation by DEPC: His-48-Ne is involved in a hydrogen bond with O $\gamma$ of Ser-18 (distance $=3.0 \mathrm{~A}$ ) and four other residues have atoms within $5.0 \AA$ from His-48-Nd, blocking access to this atom. In this context, the modified histidine either provokes a steric hindrance in the active site of the closed form or more probably prevents the formation of the closed conformation after its modification in the open state. Hence, enzyme inactivation upon modification points to an involvement of the closed conformation in the catalytic pathway of $\lambda \mathrm{L}$. A catalytic role for His-137 is ruled out as no substantial decrease in activity is observed after its mutation to Asn.

In the closed conformation, the narrowest opening at the active site entrance is reduced to approximately $2.5 \AA$ which is too small to allow the peptidoglycan substrate to enter the active site cleft. The open conformation is thus certainly involved in the catalytic pathway, at least for substrate binding. Therefore, as both conformations appear to play a role in the mechanism of action of $\lambda \mathrm{L}$, the large conformational change observed in the crystal 
structure is most likely an important functional feature.

In the case of T4L, the hinge-bending motion opens the active site cleft in the absence of substrate, and closes it upon substrate binding [8]. This active site closing was described as an intrinsic property of T4L but there was no experimental evidence for a critical role for this motion. Although the nature of the conformational change observed for $\lambda \mathrm{L}$ is different, a similar functional property may be expected as the proteins are evolutionarily related. Hence, our results support this hypothesis and suggest that closing the active site upon substrate binding is a general essential feature of viral lysozymes.

Fig. 2. Ribbon diagram illustrating the open (right) and closed (left) conformations observed in the threedimensional structure of $\lambda L$ (Protein Data Bank code lam 7 [6]). The three histidines and the essential glutamic acid (Glu-19) are represented in ball and stick mode. Only His-137 undergoes a large spatial movement upon changing from one conformation to the other.

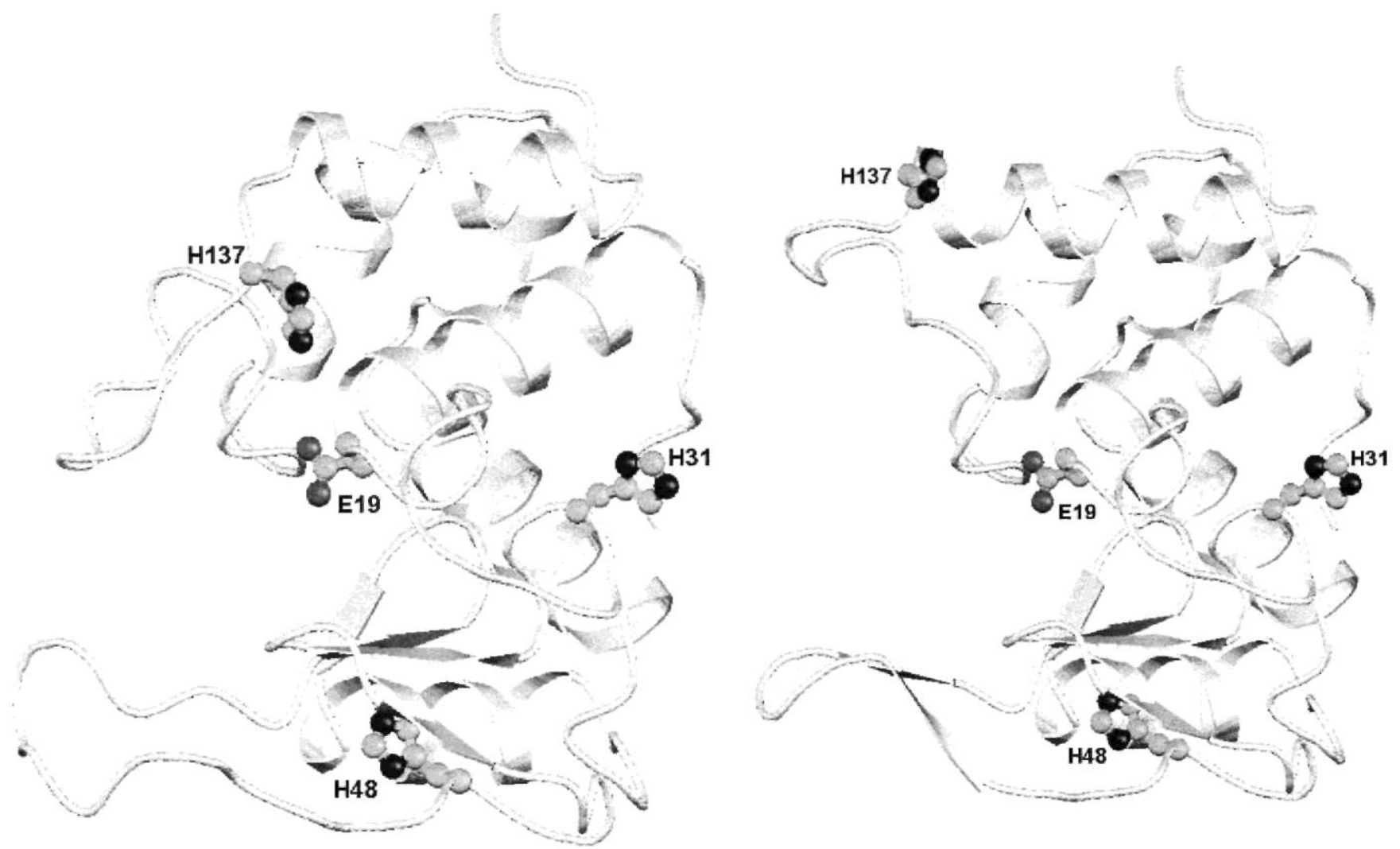

\section{Acknowledgements:}

P.S. was a Research Assistant of the Belgian National Fund for Scientific Research (FNRS). This work has benefited from a grant from the 'Fonds de Développement Scientifique' from the Université Catholique de Louvain. C.E. thanks the Fonds pour la Formation à la Recherche dans l'Industrie et l'Agriculture (FRIA) for a fellowship.

\section{References}

[1] Yon, J.M., Perahia, D. and Ghelis, C. (1998) Biochimie 80, 33-42.

[2] Imada, M. and Tsugita, A. (1971) Nature 233, 230-231.

[3] Sanger, F., Coulson, A.R., Hong, G.F., Hill, D.F. and Petersen, G.B. (1982) J. Mol. Biol. 162, 729-773. 
Published in : FEBS Letters (1999), vol. 460, pp. 442-446

Status : Postprint (Author's version)

[4] Black, L.W. and Hogness (1969) J. Biol. Chem. 244, 1968-1975, 1976-1981 and 1982-1987.

[5] Bienkowska-Szewczyk, K. and Taylor, A. (1980) Biochim. Bio-phys. Acta 615, 489-496.

[6] Evrard, C., Fastrez, J. and Declercq, J.-P. (1998) J. Mol. Biol. 276, 151-164.

[7] Jespers, L., Sonveaux, E. and Fastrez, J. (1992) J. Mol. Biol. 228, 529-538.

[8] Mchaourab, H.S., Oh, K.J., Fang, C.J. and Hubbell, W.L. (1997) Biochemistry 36, 307-316.

[9] Faber, H.R. and Matthews, B.W. (1990) Nature 348, 263-266.

[10] Zhang, X.J., Wozniak, J.A. and Matthews, B.W. (1995) J. Mol. Biol. 250, 527-552.

[11] Duewel, H., Daub, E., Robinson, V. and Honek, J.F. (1997) Biochemistry 36, 3404-3416.

[12] Imoto, T., Johnson, L.N., North, A.C.T., Phillips, D.C. and Rupley, J.A. (1972) in: The Enzymes, 3rd edn. (Boyer, P., Ed.), Vol. 7, pp. 665-868, Academic Press, New York.

[13] Tsugita, A. (1971) in: The Enzymes, 3rd edn. (Boyer, P., Ed.), Vol. 5, pp. 343-411, Academic Press, New York.

[14] Jollès, P. and Jollès, J. (1984) Mol. Cell. Biochem. 63, 165-189.

[15] Fastrez, J. (1996) EXS 75, 35-64.

[16] Taylor, A. and Gorazdowska, M. (1974) Biochim. Biophys. Acta 342, 133-136.

[17] Taylor, A., Das, B.C. and Van Heijenoort, J. (1975) Eur. J. Biochem. 53, 47-54.

[18] Bienkowska-Szewczyk, K., Lipinska, B. and Taylor, A. (1981) Mol. Gen. Genet. 184, 111-114.

[19] Engel, H., Kazemier, B. and Keck, W. (1991) J. Bacteriol. 173, 6773-6782.

[20] Thunnissen, A.M., Dijkstra, A.J., Kalk, K.H., Rozeboom, H.J., Engel, H., Keck, W. and Dijkstra, B.W. (1994) Nature 367, $750-753$.

[21] Thunnissen, A.M., Isaacs, N.W. and Dijkstra, B.W. (1995) Proteins 22, 245-258.

[22] Remaut, E., Stanssens, P. and Fiers, W. (1981) Gene 15, 81-93.

[23] Jespers, L., Sonveaux, E., Fastrez, J., Phanapoulos, A. and Davison, J. (1991) Protein Eng. 4, 485-492.

[24] Nakamaye, K.L. and Eckstein, F. (1986) Nucleic Acids Res. 14, 9679-9698.

[25] Soumillion, P. and Fastrez, J. (1992) Biochem. J. 286, 187-193.

[26] Soumillion, P. and Fastrez, J. (1995) Protein Eng. 8, 451-456.

[27] Lundblad, R.L. and Noyes, CM. (1984) in: Chemical Reagents for Protein Modification, Vol. 1, pp. 105-125, CRC Press, Boca Raton, FL.

[28] Muhlrad, A., Hegyi, G. and Toth, G. (1967) Acta Biochim. Biophys. 2, 19-29.

[29] Burstein, Y., Walsh, K.A. and Neurath, H. (1974) Biochemistry 13, 205-210.

[30] Soumillion, P. and Fastrez, J. (1998) Protein Eng. 11, 213-217. 